\title{
Numerical and Experimental Determination of Canard Controlled Missile Aerodynamic Coefficients in Subsonic Regime
}

\author{
Hamad AI KAABI, Zlatko PETROVIĆ, Gordana DJUKANOVIĆ
}

\begin{abstract}
This paper discusses the computational and experimental approach to the determination of aerodynamic coefficients of canard controlled missile with wrap around tail fin. The paper presents results of subsonic flow at Mach 0.4. Angle of attack of the missile is varied from -10 to +10 degrees with an increment of 2 degrees. Canard deflection in the pitch plane is also varied at 0,10 , and 15 degrees. The results of the simulation are compared with the experimental data obtained by the wind tunnel measurements. Measured results confirm applicability of Spalart Allmaras turbulence model with adapted computational mesh to the determination of aerodynamic coefficients for canard controlled missiles.
\end{abstract}

Keywords: aerodynamic coefficients; canard; experimental method; numerical solution; missile; subsonic flow

\section{INTRODUCTION}

Accurate determination of aerodynamic coefficients for flying vehicles is very important. They are crucial during the design phase when flight simulations are done, as well during the testing phase when flight tests are performed. Identification of missile aerodynamic coefficients is a popular field of research [1-4]. Various methods exist to determine the coefficients theoretically and experimentally. The experimental methods can be summarized into two main groups: wind tunnel and flight tests. In addition, the theoretical methods could be divided into two main groups, as well: semi-empirical methods and computational fluid dynamics methods.

Computational fluid dynamics has become a major focus due to its unique potential in identifying the aerodynamic coefficients and visualizing fluid flow. Great efforts are being put in the research on how to simulate the flow that matches the flow of the flight test in order to obtain accurate values for the aerodynamic coefficients. The research is mainly focused on the setup of the problem. This includes: generating the 3D CAD model, selecting appropriate physics of the flow, optimization of the computational procedure, as well as the estimation of computational power required to perform such computationally intensive simulations.

Employed computational technique can be separated into three major steps: optimization of the CAD model, preparation of a suitable physics setup, and running repeated simulations utilizing pressure gradient mesh adaptation until reaching the convergence of the results.

Flow about axi-symmetric canard controlled missile with wrap around tail fin is simulated using ANSYS FLUENT. Wrap around fins configurations is a major field of research [5]. The simulations are performed for several angles of attack as well as several control deflections. All simulation results are then compared with experimental data obtained from the wind tunnel experiments.

Good agreement between the CFD simulations and the experimental data for missiles with high angle of attack are found in [6]. Flow of different missile configurations was also simulated by CFD methods and agreements are achieved in $[7,8]$. Canard controlled missiles is a major field of research in [9] and [10]. However, it is very rare to find simulations for canard controlled missiles with the deflection of control surfaces as well as high angle of attack.

The flow for Mach number 0.4 and for the angle of attack varying from -10 to 10 degrees is calculated in order to analyze the missile's static stability. Canard control deflections are also simulated up to 15 degrees to capture the non-linearity as well as controllability and maneuver capabilities of the missile. Exact conditions are replicated in the wind tunnel with full scale model to assess accuracy and quality of calculated solution.

\section{MODEL PREPARATION}

Missile model used in simulations is prepared using commercial modelling software package, CATIA. Model is identical to the real model used in the wind tunnel testing. Fig. 1 illustrates the model used in simulations including all the control surfaces.

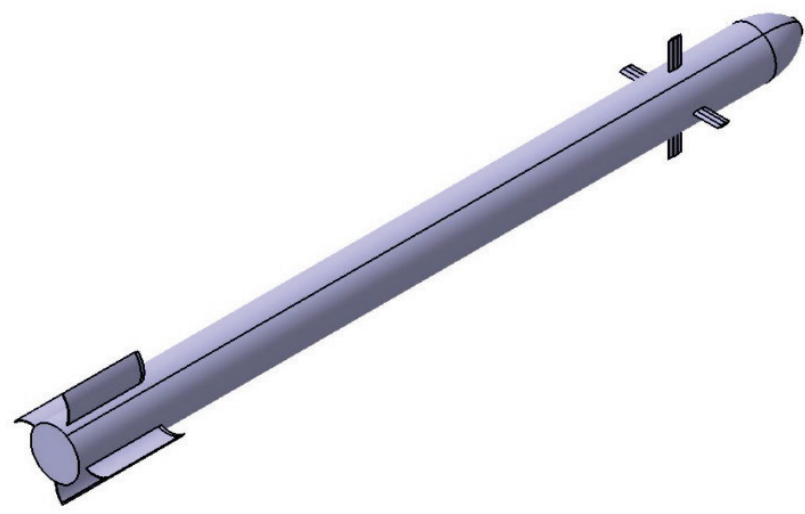

Figure $1 \mathrm{CAD}$ model of the missile

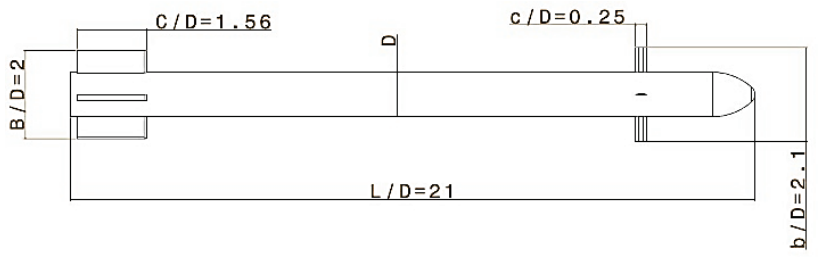

Figure 2 Proportions of the missile

Principal technical drawing of the CAD model presented previously is shown in Fig. 2 where relative dimensions to the missile caliber are shown. 
Due to the large amount of tiny details, it was necessary to simplify the model and avoid unnecessary complications in mesh generation. Model is simplified by neglecting these details without sacrificing the important missile features.

Among the whole missile parts, canards proved to have the greatest effect on both the mesh size as well as the final results. Fig. 3 shows canard cross section which includes all manufactured smoothings and roundings.

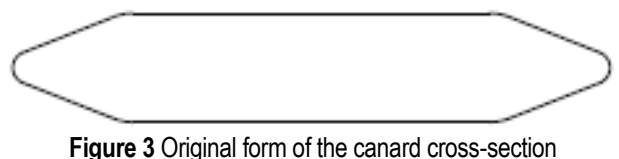

Idealized model is generated from the real model by ignoring all roundings and smoothings as it is shown in Fig. 4, this simplification reduces cross-sectional shape to six line segments. Introduced simplifications do not have great impact on calculated results which is confirmed later in this paper.

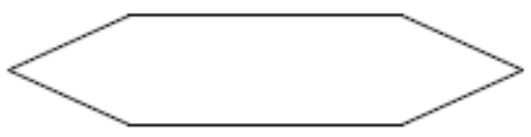

Figure 4 Simplified cross-section of the canards

\subsection{Simulation Setup}

Flow simulation requires proper specification of boundary conditions, size of the computational grid as well as position of boundary surfaces, and selection of the proper simulation flow model. Pressure based solver is used for all the simulations.

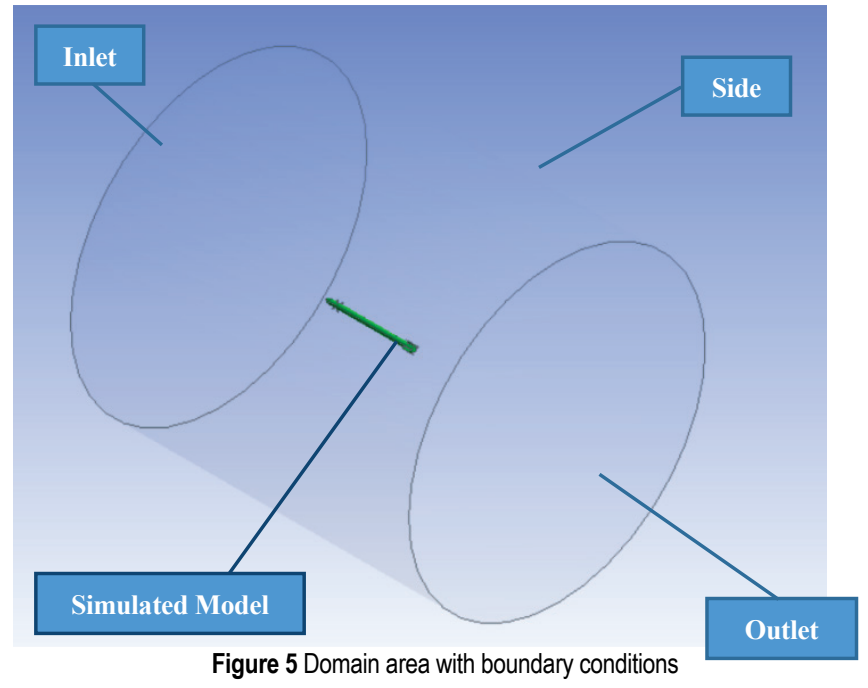

The computational domain is shaped like a cylinder. Inlet, outlet and sides outer surfaces are placed far enough so that computed results are not influenced by their position. The distance from the inlet to the nose of the missile is 1 times the length of the model while the distance from the back of the missile to the outlet is 5 times length of the missile. The radius of the computational cylinder is 5 times length of the missile.

The boundary conditions are required to solve the mathematical model by specifying the fluid properties, velocity direction and magnitude over boundary surface. In the simulation, the boundary conditions are inlet, outlet, and sides described by a cylindrical flow domain as shown in Fig. 5 (Note: domain area is scaled down to show simulated model clearly), as well as impermeability condition of the missile surface and zero velocity (both normal and tangential components).

All angles of attack are simulated by specifying the flow direction on all outer boundary surfaces. Components of the flow are defined according to the sine and cosine of angle of attack.

The Inlet, sides, as well as outlet were set as pressure farfield with the following boundary conditions

Table 1 Boundary conditions parameters values

\begin{tabular}{|c|c|}
\hline Parameter & Value \\
\hline$M$ & 0.4 \\
\hline$p$ & $90748 \mathrm{~Pa}$ \\
\hline$T$ & $288.76 \mathrm{~K}$ \\
\hline$v$ & 10 \\
\hline
\end{tabular}

\subsection{Meshing}

The mesh is prepared in such a way to include all the details of the simulated model without considering the flow disturbances such as control deflection or angle of attack. This means the mesh will be simple and does not have areas with numerous mesh polygons to capture flow disturbances. Hence, the baseline mesh is identical for all the simulations cases such as varying angle of attack and control deflections. The baseline mesh statistics is presented in Tab. 2

Table 2 Baseline mesh statistics

\begin{tabular}{|l|c|}
\hline \multicolumn{1}{|c|}{ Parameter } & Value \\
\hline Number of nodes & 211043 \\
\hline Number of elements & 1162516 \\
\hline
\end{tabular}

The simulated model parts surfaces are meshed separately using face sizing with unique element size as shown in Tab. 3.

Table 3 Model parts mesh elements size
\begin{tabular}{|l|c|}
\hline \multicolumn{1}{|c|}{ Model part } & Element size $(\mathrm{mm})$ \\
\hline Body & 10 \\
\hline Nose & 4 \\
\hline Tail fin & 3 \\
\hline
\end{tabular}

There is no need to apply face size mesh to the canards due to their simplified shape. In addition, the canards face mesh will be improved later in this paper.

\subsection{Air Flow Characteristics}

The simulated fluid is selected to be air with ideal gas characteristics. Sutherland's law is used for viscosity calculations. Three Coefficient Method of Sutherland's law is used with the parameters presented in Tab. 4

\begin{tabular}{|l|c|}
\multicolumn{1}{|c|}{ Table 4 Sutherland's law } \\
\begin{tabular}{|l|c|}
\hline \multicolumn{1}{|c|}{ Parameter } & Value \\
\hline Reference viscosity & $1.716 \mathrm{e}-05 \mathrm{~kg} /(\mathrm{m} \cdot \mathrm{s})$ \\
\hline Reference temperature & $273.11 \mathrm{~K}$ \\
\hline Effective temperature & $110.56 \mathrm{~K}$ \\
\hline
\end{tabular}
\end{tabular}




\subsection{Steady State Flow}

The type of the flow simulation is selected according to the flow simulation conditions. Most of the simulations performed assumed a steady state flow. However, there are some simulations where transient flow is assumed, such as the simulations with missile high angle of attack and large control deflections.

\subsection{Turbulence Model}

Fundamental equations governing the fluid flow are decomposed into mean and turbulent fluctuation terms and closed by additional equations. Simulations are performed using One-Equation Spalart Allmaras turbulence model that utilizes the following transport Eq. (1) [11].

$$
\begin{aligned}
& \frac{D \tilde{v}}{D t}=C_{\mathrm{b} 1}\left(1-f_{t 2}\right) \tilde{S} \tilde{v}+\frac{1}{\sigma}\left[\nabla(v+\tilde{v}) \nabla \tilde{v}+C_{\mathrm{b} 2}(\nabla \tilde{v})^{2}\right]- \\
& -\left[C_{w 1} f_{\mathrm{w}}-\frac{C_{\mathrm{b} 1}}{\kappa^{2}} f_{\mathrm{t} 2}\right]\left(\frac{\tilde{v}}{d}\right)^{2}+f_{\mathrm{t} 1} \Delta U^{2}
\end{aligned}
$$

The standard values of model coefficients are listed in Tab. 5.

Table 5 Spalart Allmaras coefficients used in simulation

\begin{tabular}{|c|c|}
\hline Model Constant & Value \\
\hline$C_{\mathrm{b} 1}$ & 0.135 \\
\hline$C_{\mathrm{b} 2}$ & 0.622 \\
\hline$C_{\mathrm{v} 1}$ & 7.1 \\
\hline$C_{\mathrm{w} 2}$ & 0.3 \\
\hline$C_{\mathrm{w} 3}$ & 2.0 \\
\hline
\end{tabular}

Spalart Allmaras turbulence model is used in this research for its simplicity, reasonable accuracy, stability and speed of computation.

\subsection{Mesh Adaptation}

Several available techniques for mesh adaptation in FLUENT are used, such as the gradient and the geometrybased adaptations. They are used to either refine or course the mesh based on the simulation solution or the geometry of the problem.

All the simulations meshes are modified and improved by the pressure gradient method which is a built-in function in ANSYS FLUENT. This method utilizes the pressure Euclidean norm along with a characteristics length scale according to Eq. (2) [12].

$\left|\mathrm{e}_{\mathrm{i} 1}\right|=\left(A_{\text {cell }}\right)^{\frac{r}{2}}|\nabla f|$

The process of mesh adaption in the simulation is shown in Fig. 6. Fig. 6 shows the cells that require refinement, in red, according to the pressure gradient criteria. Moreover, an angle of attack of 10 degrees is used to show how the pressure gradients are following the angled flow direction according to the yellow box. It is important to point out that the mesh polygons increased from around 1.1 million cells to almost 4 million cells with slight variance depending on each flow condition.

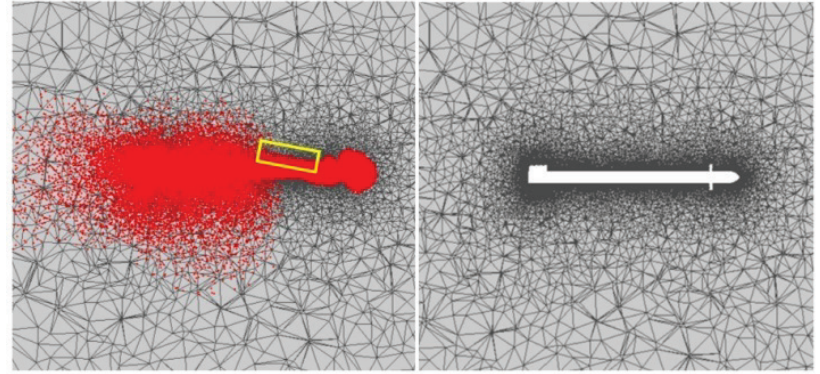

Figure 6 Mesh adaptation process

Several simulations are performed for different cases of control deflections as well as angles of attack

\begin{tabular}{|l|c|c|}
\multicolumn{1}{|c}{ Table 6 Simulated cases } \\
\hline & Angle of Attack & Pitch Deflection \\
\hline Range & -10 to 10 degrees & $0,10,15$ degrees \\
\hline Step Interval & 2 degrees & N/A \\
\hline
\end{tabular}

Two of the four canards are used for pitch control. Pitch control canard's deflection sign convention is shown in Fig. 7. Fig. 7 shows the view from the rear of the model. In addition, the lowered edges of the deflected panels are the trailing edges.

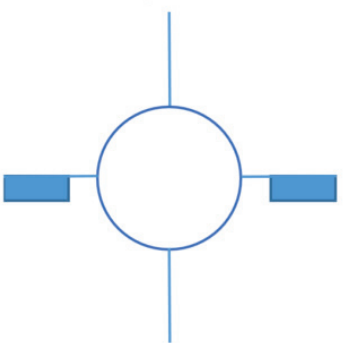

Figure 7 Rear view of the positive pitch control deflection

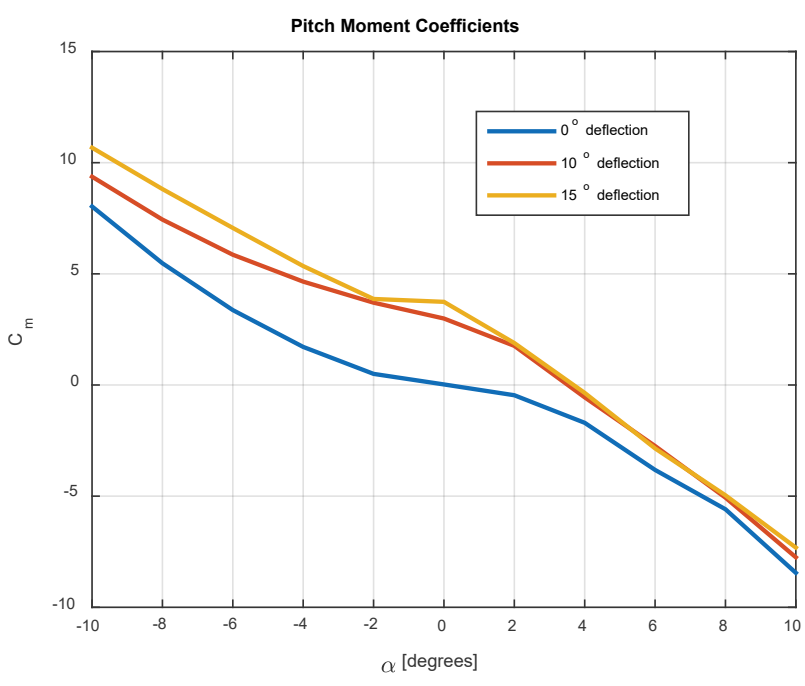

Figure 8 Pitch moment coefficients

\section{RESULTS}

\subsection{Pitching Moment Coefficients}

The pitching moment coefficients are crucial to analyze static stability of the model as well as the maneuver and control capabilities. Therefore, several simulations are performed to include the pitch canard control deflection up to 15 degrees. This allows for the comprehensive study of the full range of deflection capability. Moreover, it allows 
for the identification of the maximum control deflection angle without losing maneuver capability. It is important to point out that the moments are measured about a reference point located at $42 \%$ of the model length away from the model's nose tip.

Fig. 8 shows the simulation data for pitch moment coefficient. It is important to notice that the canard control deflection of 10 and 15 degrees is almost identical near higher positive angle of attack which is a clear indication that canards lose effectiveness when approaching 15 degrees.

\subsection{Normal Force Coefficient}

The lift coefficient can be directly calculated from the normal force coefficient. The lift coefficient is crucial when it comes to evaluating the missile flight performance parameters such as stall speed and maneuver capability. The results of the normal force coefficients obtained in the simulation are shown in Fig. 9. It is important to point out that the missile starts to lose lift force when deflection approaches 15 degrees for almost all positive angle of attack.

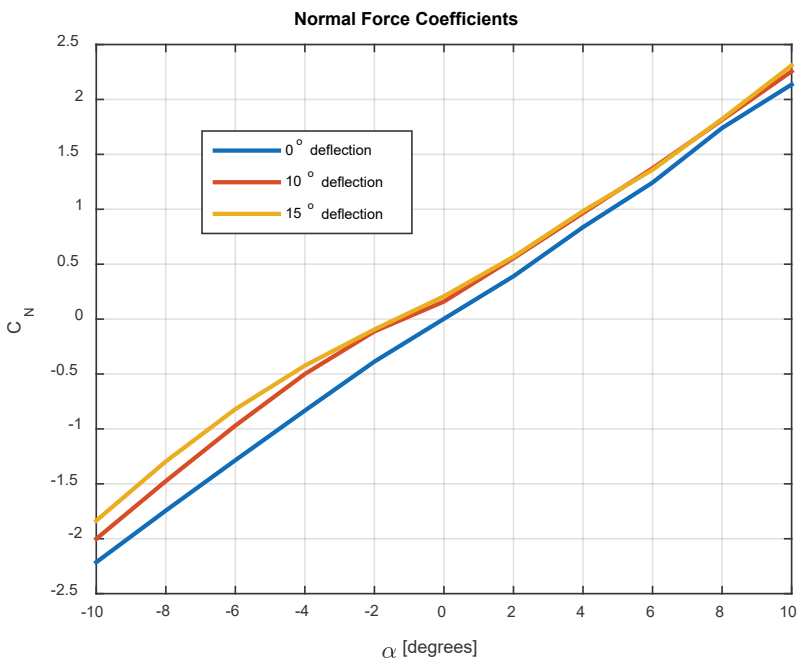

Figure 9 Normal force coefficients

By comparing Fig. 8 and Fig. 9, it can be seen that both the pitching moment and the normal force coefficients lose performance at 15 degrees canard control deflection. Therefore, the canards control effectiveness fades away when approaching 15 degrees control deflection as well as high angle of attack on the same plane. This conclusion is supported in the following section where flow contours are presented.

\subsection{Mach Number Contours}

Once the simulation is completed, contours of all flow variables can be presented. Nevertheless, the Mach number contours are selected for illustration and further analysis. More specifically, the cases of 0 and 10 degrees angle of attack are chosen since they demonstrate the major flow characteristics.

The angle of attack 0 degrees with control deflection of 0 degrees is presented in Fig. 10. As expected, the flow is mainly axisymmetric except in the vicinity of the wrap around tail fins.

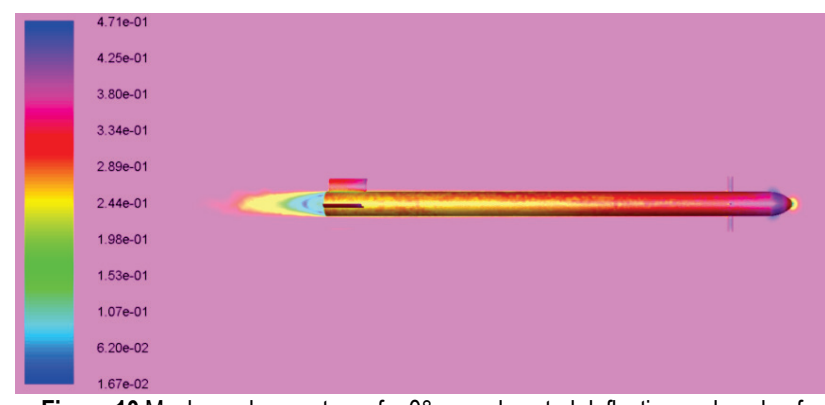

Figure 10 Mach number contours for $0^{\circ}$ canard control deflection and angle of attack $\alpha=0^{\circ}$

The angle of attack 10 degrees with control deflection of 0 degrees is shown in Fig. 11. Here, the angle of attack effect is clearly seen behind the canard section. However, there is no extraordinary behavior expected from the two cases due to pitch canard control deflection of 0 degrees.

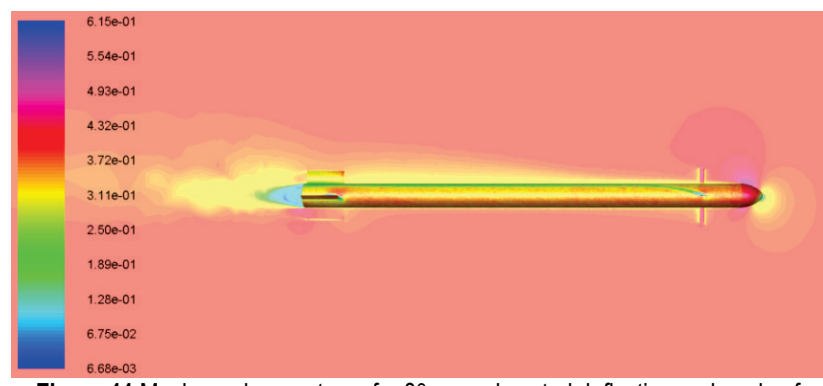

Figure 11 Mach number contours for $0^{\circ}$ canard control deflection and angle of attack $\alpha=10^{\circ}$

The same simulation cases are repeated but with 10 degrees pitch canard control deflection. This allows the analysis of canard control effectiveness. The angle of attack 0 degrees with control deflection of 10 degrees is shown in Fig. 12. It is important to point out that the effect of the canard control is clear in Fig. 12 when comparing with Figure 10 for zero control deflection.

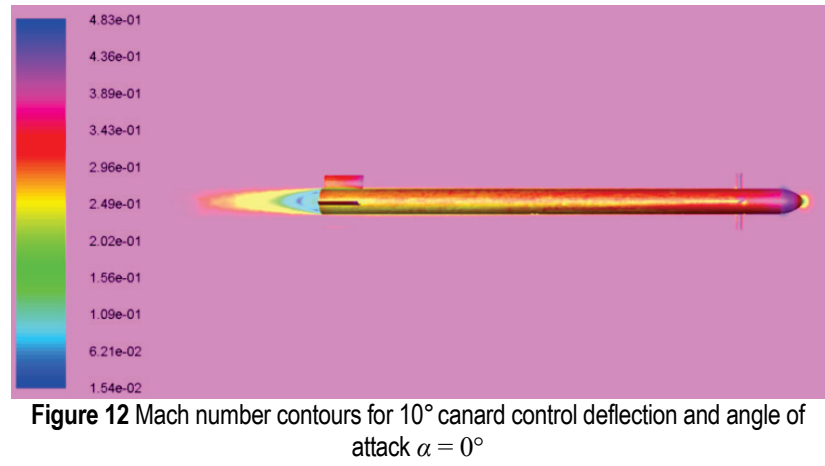

The angle of attack 10 degrees with control deflection of 10 degrees is shown in Fig. 13. Flow separation starts to increase in Fig. 13 when both angle of attack and control deflection are 10 degrees. That means the canard angle of attack is approximately 20 degrees.

This section includes simulations with the highest pitch canard control deflection of 15 degrees for both 0 and 10 degrees angle of attack. These simulations are crucial to ensure the limit of the canard control effectiveness. Fig. 14 shows the angle of attack 0 degrees with control deflection of 15 degrees. 


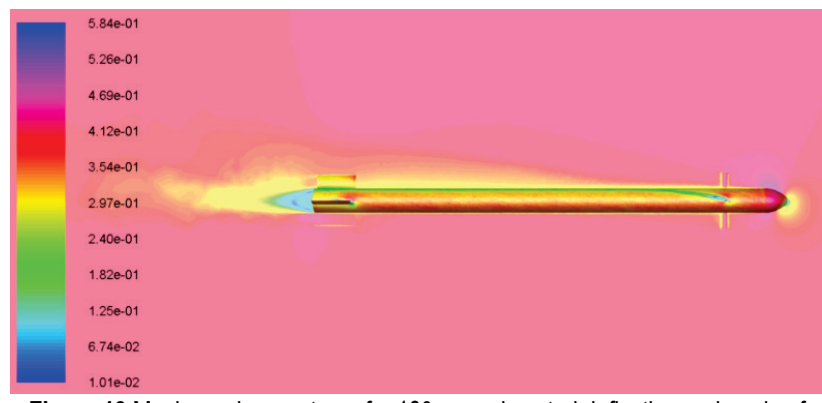

Figure 13 Mach number contours for $10^{\circ}$ canard control deflection and angle of attack $\alpha=10^{\circ}$

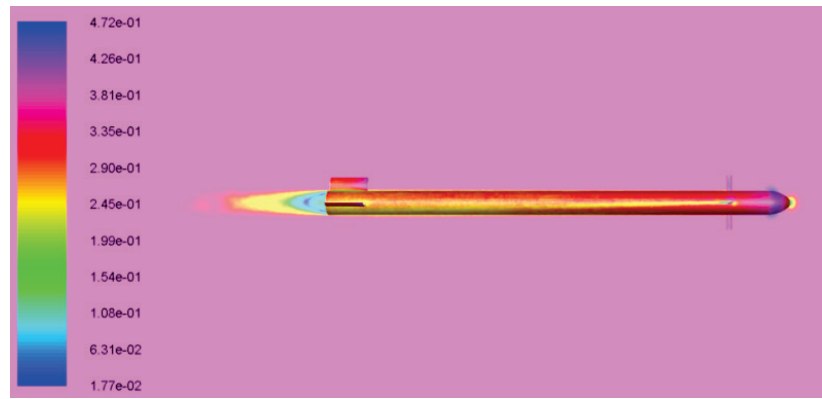

Figure 14 Mach number contours for $15^{\circ}$ canard control deflection and angle of attack $\alpha=0^{\circ}$

Fig. 15 shows the angle of attack 10 degrees with control deflection of 15 degrees. It is important to note the clear transient flow separation which resulted from the summation of both angles resulting in 25 degrees flow inclination towards the canards.

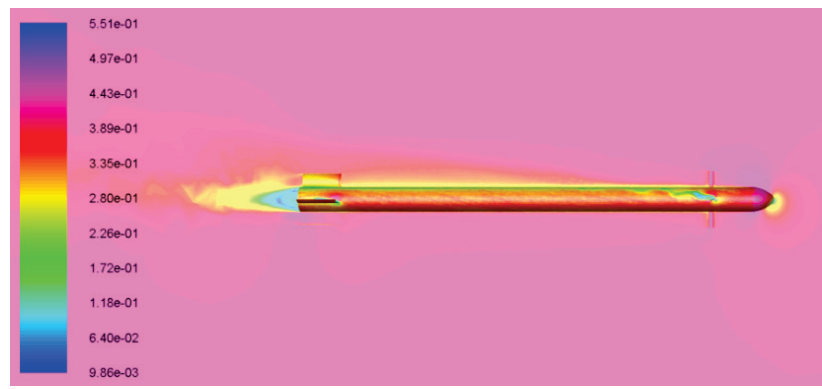

Figure 15 Mach number contours for $15^{\circ}$ canard control deflection and angle of attack $\alpha=10^{\circ}$

\subsection{Wind Tunnel Testing}

The Experimental Aerodynamics Laboratory in Military Technical Institute is one of the major aerodynamics laboratories in Belgrade, Serbia. The laboratory allows testing of both aircraft and missile models. It comprises five wind tunnels for different purposes.

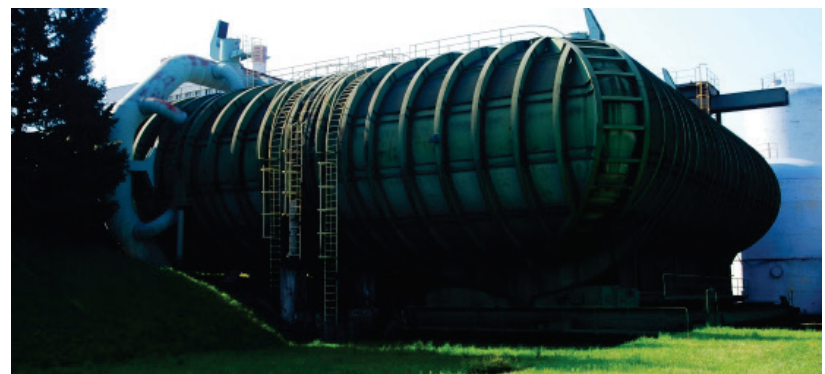

Figure 16 External view of the T-35 wind tunnel
The physical prototype is tested in the T-35 wind tunnel, which is a large closed-circuit wind tunnel. The T-35 wind tunnel has the capability of testing the flow from Mach 0.1 to 0.5 . The external view of the T-35 wind tunnel is shown in Fig. 16 [13]

In addition, the T-35 wind tunnel is capable of testing full scale models that can fit in $4.4 \times 3.2 \mathrm{~m}$ test section. Therefore, the full model is tested without any scaling.

Full scale model was manufactured and inspected by 3D scanning to assure the quality of production. The control deflections of the canards are adjusted by screws with different sizes according to the deflection degree. Angle of attack is varied by the moving stinger which changes the whole orientation of the model with respect to the flow direction. A view of the model body in the test section is provided in Fig. 17.

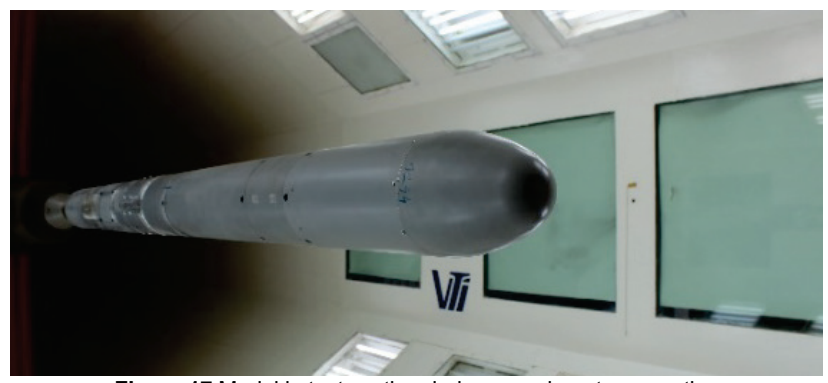

Figure 17 Model in test section during experiment preparation

All aerodynamic coefficients were collected together with the consistent angle of attack measured by the lab sensors in the stinger. The whole testing lasted nearly one week in time to cover all the testing configurations. Although each measurement took almost 1 minute, the preparation for each experimental set-up took most of the time.

\section{DISCUSSION}

After testing the physical prototype model in the wind tunnel with the same simulated conditions, the aerodynamic coefficient data are compared for all control deflections and angle of attack cases.

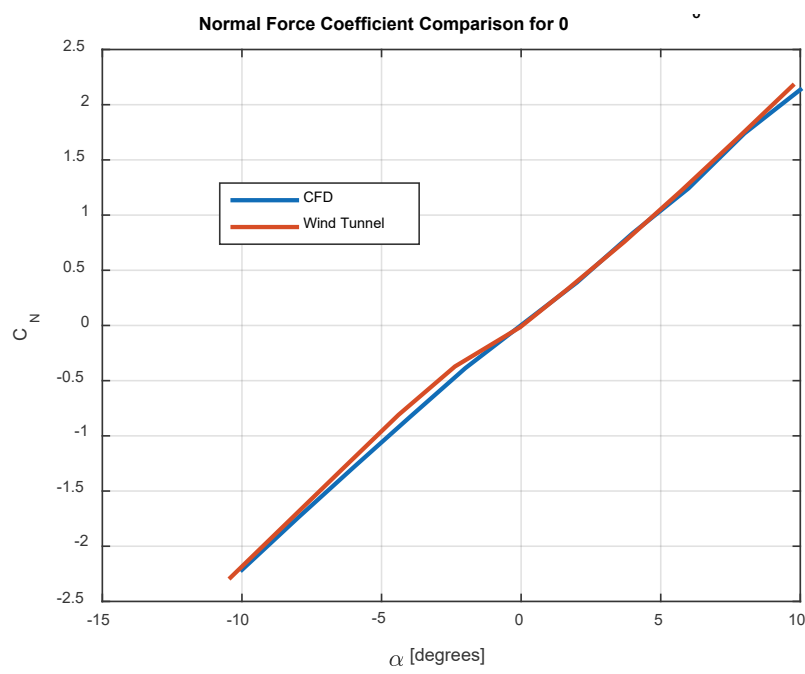

Figure 18 Normal force coefficient comparison for $0^{\circ}$ canard control deflection

The following cases represent the normal force coefficient as well as the pitching moment coefficient for 0 
degrees pitch canard control deflection as shown in Fig. 18 and Fig. 19. The zero canard control deflection results from both wind tunnel and CFD simulation are almost identical for both pitch moment coefficient as well as normal force coefficient.

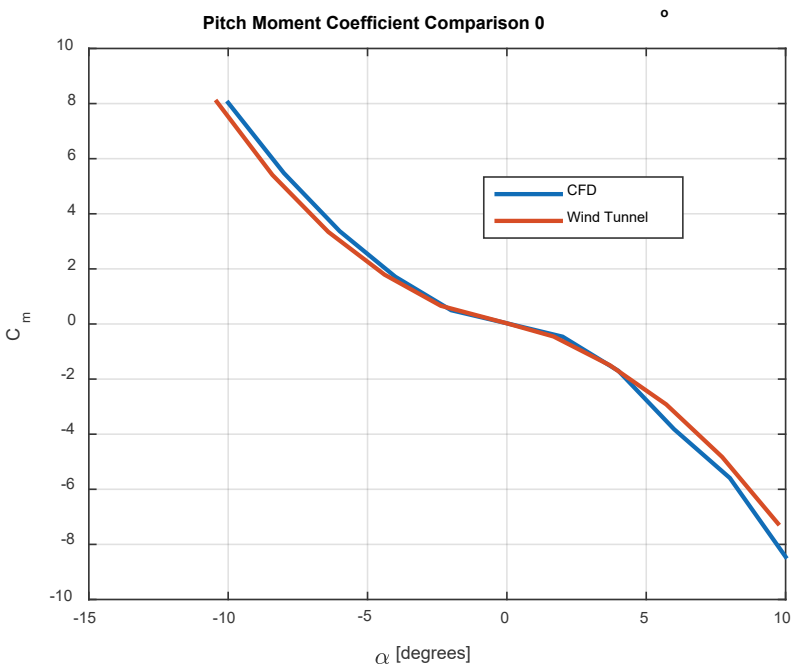

Figure 19 Pitch moment coefficient comparison for $0^{\circ}$ canard control deflection

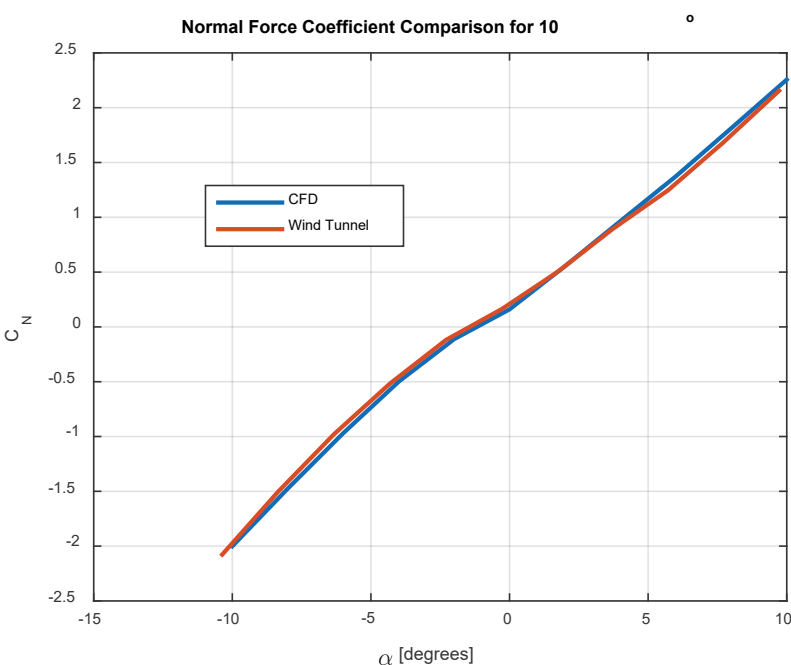

Figure 20 Normal force coefficient comparison for $10^{\circ}$ canard control deflection

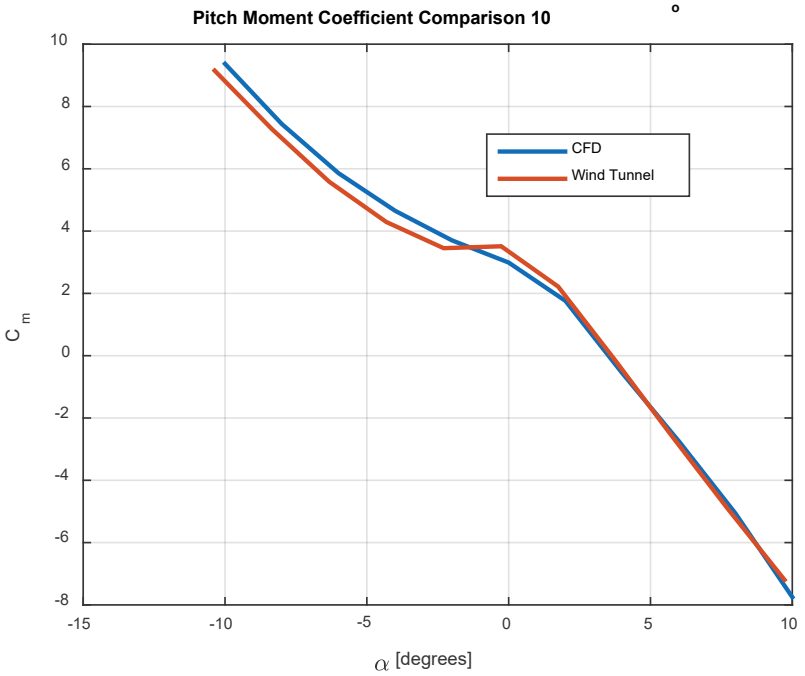

Figure 21 Pitch moment coefficient comparison for $10^{\circ}$ canard control deflection
The following cases represent the normal force coefficient as well as the pitching moment coefficient for 10 degrees pitch canard control deflection as shown in Fig. 20 and Fig. 21. It can be seen that the results from both wind tunnel and CFD simulations show great consistency even at high angle of attack.

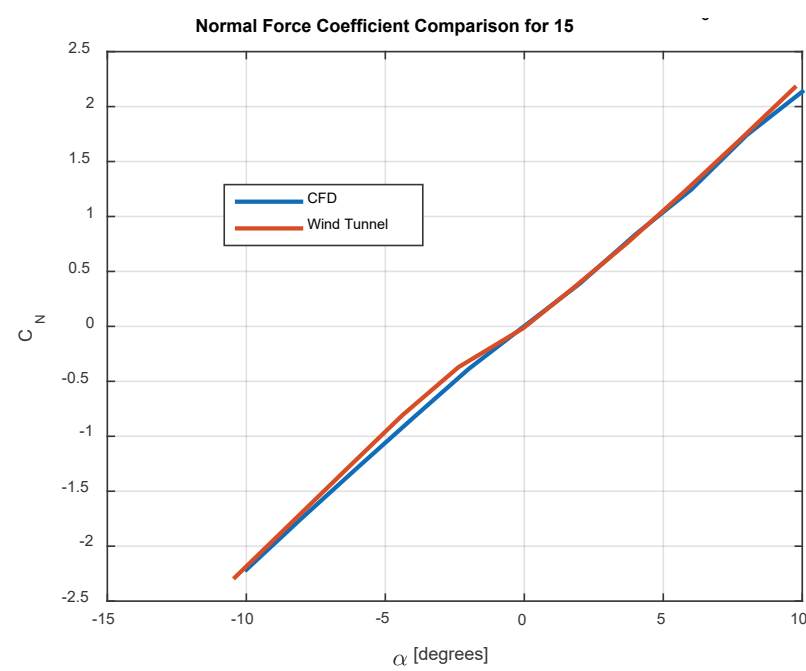

Figure 22 Normal force coefficient comparison for $15^{\circ}$ canard control deflection

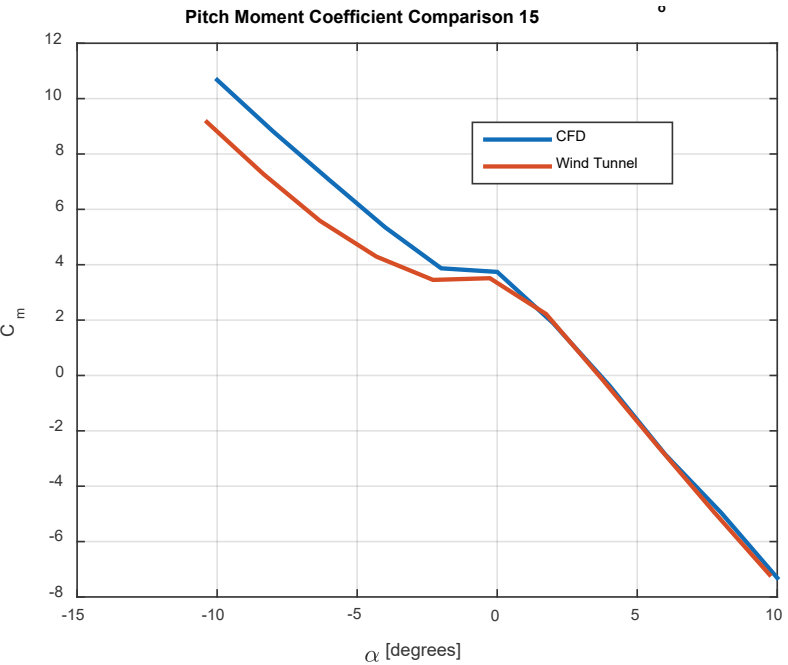

Figure 23 Pitch moment coefficient comparison for $15^{\circ}$ canard control deflection

The following cases represent the normal force coefficient as well as the pitching moment coefficient for 15 degrees pitch canard control deflection as shown in Fig. 22 and Fig. 23. It can be seen that the normal force coefficient from CFD simulation is in extreme agreement with the wind tunnel data. In addition, the same agreement is obtained for pitch moment coefficient but with slight deviation towards high negative angle of attack as seen in Fig. 23.

\section{CONCLUSIONS}

This paper presented and discussed a successful numerical approach by finite volume method mainly realized by improving the mesh utilizing pressure gradient methods. As seen in the paper, the CFD simulation results showed a good agreement with experimental results performed in the wind tunnel for Mach 0.4. Maximum deviation occurred for pitching moment coefficient at 15 degrees. However, the error was less than $20 \%$. Therefore, 
Spalart Allmaras turbulence model along with Sutherland law can be considered accurate for subsonic flow regime.

Employed CFD approach proved numerous advantages even over the wind tunnel testing. It provided much more insights in the flow field. Numerical simulation is much faster and more economic than wind tunnel testing given the right computational resources. It can also reduce the cost of testing different configurations in the wind tunnel. However, wind tunnel is always needed to validate the results since CFD has limitations on certain flow conditions (such as flow separation, transient flow, transonic flow, etc.) as well as certain simulated models.

In addition, the results also showed that the flow around the canards started to separate when applying 15 degrees control deflection simultaneously with angle of attack 10 degrees. Therefore, pitch canard control surfaces may not be as effective when deflecting more than 15 degrees. Since obtained numerical and experimental data correlate sufficiently in subsonic flow regime, the study will be further extended to transonic and supersonic velocities.

\section{Acknowledgements}

I would like to extend my appreciation to Professor Danilo Cuk for his continuous support throughout my graduate education journey.

I take this opportunity to express my gratitude to the professors of the Faculty of Mechanical Engineering at the University of Belgrade, as well as the Emirates Advanced Research Technology Holdings for their support.

\section{REFERENCES}

[1] Kim, D. \& Lee, H. (2018). Practical Applications of a Building Method to Construct Aerodynamic Database of Guided Missile Using Wind Tunnel Test Data. International Journal of Aeronautical and Space Sciences, 19(1), 1-12. https://doi.org/10.1007/s42405-018-0017-3

[2] Peng, J., Zhao, L., \& Jiao, L. (2016). Numerical Simulations on Aerodynamic Characteristics of a Guided Rocket Projectile. Manufacturing Technology and Control, $3^{\text {rd }}$ International Conference on Materials Engineering / Taiyuan, 1004-1007. https://doi.org/10.2991/icmemtc-16.2016.199

[3] De Spirito, J. (2003). Subsonic Flow CFD Investigation of Canard-Controlled Missile with Planar and Grid Gins. AIAA, 27, 1-13. https://doi.org/10.2514/6.2003-27

[4] Decrocq, C., Martinez, B., Albisser, M., Dobre, S., Gnemmi, P., Bailly, Y., \& Roy, J. (2017). Aerodynamic Prediction of a Projectile Fitted With Fins. International Conference on Applied Aerodynamics / Lyon, 1-11. https://doi.org/10.1108/HFF-06-2017-0259

[5] Chen, H., Li, K., Wang, C., Xia, F., Li, B., Wang, S., Deng, H., Zhai, R., \& Xia, Z. (2017). Analysis of Aerodynamic Characteristics of a Modular Assembled Missile with Canard Rudder and Arc Tail. Identification and Control, the $9^{\text {th }}$ International Conference of Modelling / Kumming, 976-981. https://doi.org/10.1109/ICMIC.2017.8321598

[6] Srivastava, B. (1991). Lateral jet control of a supersonic missile - CFD predictions and comparison to Force and moment measurements. AIAA Paper 97-0639, 1-45

[7] Kumar, R., Qumar, M. S., \& Vishak T. (2017). Studies on Aircraft Store with Rotating Tail. 55th AIAA Aerospace Sciences Meeting, AIAA SciTech Forum / Grapevine, Texas, 1-8. https://doi.org/10.2514/6.2017-0270

[8] Allen, J. \& Mehdi, G. (2018). Forced Motions Design for Aerodynamic Identification and Modeling of a Generic
Missile Configuration. Aerospace Science and Technology, 77, 742-754. https://doi.org/10.1016/j.ast.2018.04.014

[9] Chen, Y., Gao, X., Gao, M., \& Lv, H. (2017). Aerodynamic Characteristics of a Canard Guided Rocket. World Scientific, 8(1), 1750001-1-1750001-22. https://doi.org/10.1142/S1793962317500015

[10] Sahu, J. \& Fresconi, F. (2017). Computational and Experimental Free-Flight Motion of a Subsonic CanardControlled Body. AIAA Aviation Forum, 35 th AIAA Applied Aerodynamics Conference / Denver, 1-19. https://doi.org/10.2514/6.2017-3400

[11] Spalart, P. R. \& Allmaras, S. R. (1992). A One-Equation Turbulence Model for Aerodynamic Flows. AIAA, 92-0439, 127. https://doi.org/10.2514/6.1992-439

[12] (2009). ANSYS FLUENT 12.0 Theory Guide. ANSYS Inc.

[13] Laboratories of the Military Technical Institute. http://www.vti.mod.gov.rs/index.php?view=labs\&id=4/ (Accessed: 20.9.2017)

\section{Contact information:}

Hamad AI KAABI, MSc

University of Belgrade,

Faculty of Mechanical Engineering Belgrade,

Kraljice Marije 16, 11120, Belgrade 35, Serbia

alkaabih999@gmail.com

\section{Zlatko PETROVIC, Professor, Dr \\ University of Belgrade, \\ Faculty of Mechanical Engineering Belgrade, \\ Kraljice Marije 16, 11120, Belgrade 35, Serbia \\ zpetrovic@mas.bg.ac.rs}

Gordana DJUKANOVIC, Assistant Professor, Dr

University of Belgrade,

Faculty of Forestry Belgrade,

Kneza Viseslava 1, 11030 Belgrade, Serbia 